\title{
Non-perturbative effects in the electro-weak theory versus TEVATRON and LHC data
}

\author{
Boris Arbuzov* \\ SINP MSU \\ E-mail: arbuzov@theory.sinp.msu.ru
}

\begin{abstract}
We apply Bogoliubov compensation principle to the gauge electro-weak interaction. The nontrivial solutions of compensation equations lead to determination of parameters of the theory including value of gauge electro-weak coupling $g\left(M_{W}^{2}\right) \simeq 0.62$ and the $t$-quark mass $m_{t}=177 \mathrm{GeV}$ in satisfactory agreement with the experimental values. The results strongly support idea of $\bar{t} t$ condensate as a source of the electro-weak symmetry breaking. The approach also gives predictions for experiments at LHC and TEVATRON. In particular, very large mass of the composite Higgs boson is obtained, which means prediction of a negative result of Higgs boson searches at LHC. The indications (CDF) for state with mass $\simeq 145 \mathrm{GeV}$ are interpreted as a manifestation of $I=1, J=1$ bound state of two $W$. Corresponding cross-sections and decay widths are estimated.
\end{abstract}

The XXth International Workshop High Energy Physics and Quantum Field Theory September 24 - October 1, 2011

Sochi, Russia

${ }^{*}$ Speaker. 


\section{Compensation equation for anomalous tree-boson interaction}

In works [1, 2, 3, 4, 5, 6] N.N. Bogoliubov compensation principle was applied to studies of spontaneous generation of effective non-local interactions in renormalizable gauge theories.

N.N. Bogoliubov compensation principle [7, 8, 9] was very succesfully applied to non-perturbative problems in ststistical physics (superfluidity, supercoductivity etc. The first application of Bogoliubov compensation principle to problems of the quantum field theory see [10].

At the present time quite important problem of QFT consists in studying of a spontaneous generation of effective theories. The compensation approach allows to check if an effective interaction could be generated in a chosen variant of a renormalizable theory. In view of this one performs "add and subtract" procedure for the effective interaction with a form-factor. Then one assumes the presence of the effective interaction in the interaction Lagrangian and the same term with the opposite sign is assigned to the newly defined free Lagrangian.

We start with EW Lagrangian with 3 lepton and quarks with gauge group $S U(2)$.

$$
\begin{aligned}
& L=\sum_{k=1}^{3}\left(\frac{l}{2}\left(\bar{\psi}_{k} \gamma_{\mu} \partial_{\mu} \psi_{k}-\partial_{\mu} \bar{\psi}_{k} \gamma_{\mu} \psi_{k}\right)+\frac{g}{2} \bar{\psi}_{k L} \gamma_{\mu} \tau^{a} W_{\mu}^{a} \psi_{k L}\right)+ \\
& +\sum_{k=1}^{3}\left(\frac{l}{2}\left(\bar{q}_{k} \gamma_{\mu} \partial_{\mu} q_{k}-\partial_{\mu} \bar{q}_{k} \gamma_{\mu} q_{k}\right)+\frac{g}{2} \bar{q}_{k L} \gamma_{\mu} \tau^{a} W_{\mu}^{a} q_{k L}\right)- \\
& -\frac{1}{4}\left(W_{\mu \nu}^{a} W_{\mu \nu}^{a}\right) ; \quad W_{\mu \nu}^{a}=\partial_{\mu} W_{v}^{a}-\partial_{\nu} W_{\mu}^{a}+g \varepsilon_{a b c} W_{\mu}^{b} W_{v}^{c} .
\end{aligned}
$$

where we use the standard notations.

In accordance to the Bogoliubov approach in application to QFT we look for a non-trivial solution of a compensation equation, which is formulated on the basis of the Bogoliubov procedure add - subtract.

$$
\begin{aligned}
& L=L_{0}+L_{i n t} ; \\
& L_{0}==\sum_{k=1}^{3}\left(\frac{\imath}{2}\left(\bar{\psi}_{k} \gamma_{\mu} \partial_{\mu} \psi_{k}-\partial_{\mu} \bar{\psi}_{k} \gamma_{\mu} \psi_{k}\right)-m_{k} \bar{\psi}_{k} \psi_{k}+\frac{\imath}{2}\left(\bar{q}_{k} \gamma_{\mu} \partial_{\mu} q_{k}-\partial_{\mu} \bar{q}_{k} \gamma_{\mu} q_{k}\right)-\right. \\
& \left.-M_{k} \bar{q}_{k} q_{k}\right)-\frac{1}{4} W_{\mu \nu}^{a} W_{\mu \nu}^{a}+\frac{G}{3 !} \cdot \varepsilon_{a b c} W_{\mu \nu}^{a} W_{v \rho}^{b} W_{\rho \mu}^{c} ; \\
& L_{i n t}=\frac{g}{2} \sum_{k=1}^{3}\left(\bar{\psi}_{k} \gamma_{\mu} \tau^{a} W_{\mu}^{a} \psi_{k}+\bar{q}_{k} \gamma_{\mu} \tau^{a} W_{\mu}^{a} q_{k}\right)-\frac{G}{3 !} \cdot \varepsilon_{a b c} W_{\mu \nu}^{a} W_{v \rho}^{b} W_{\rho \mu}^{c} .
\end{aligned}
$$

Here isotopic summation is performed inside of each quark bi-linear combination, and notation $-\frac{G}{3 !} \cdot \varepsilon_{a b c} W_{\mu \nu}^{a} W_{\nu \rho}^{b} W_{\rho \mu}^{c}$ means corresponding non-local vertex in the momentum space

$$
\begin{aligned}
& (2 \pi)^{4} G \varepsilon_{a b c}\left(g_{\mu v}\left(q_{\rho} p k-p_{\rho} q k\right)+g_{v \rho}\left(k_{\mu} p q-q_{\mu} p k\right)+g_{\rho \mu}\left(p_{v} q k-k_{v} p q\right)+\right. \\
& \left.+q_{\mu} k_{v} p_{\rho}-k_{\mu} p_{v} q_{\rho}\right) F(p, q, k) \delta(p+q+k)+\ldots ;
\end{aligned}
$$

where $F(p, q, k)$ is a form-factor and $p, \mu, a ; q, v, b ; k, \rho, c$ are respectfully incoming momenta, Lorentz indices and weak isotopic indices of $W$-bosons. We mean also that there are present fourboson, ... 
Effective interaction (4.1) is usually called anomalous three-boson interaction and it is considered for long time on phenomenological grounds [11]. Note, that the first attempt to obtain the anomalous three-boson interaction in the framework of Bogoliubov approach was done in work [12]. Our interaction constant $G$ is connected with conventional definitions in the following way

$$
G=-\frac{g \lambda}{M_{W}^{2}}
$$

The current limitations for parameter $\lambda$ read [13]

$$
\lambda=-0.016_{-0.023}^{+0.021} ; \quad-0.059<\lambda<0.026(95 \% \text { C.L. }) .
$$

Due to our approximation $\sin ^{2} \theta_{W} \ll 1$ we use the same $M_{W}$ for both charged $W^{ \pm}$and neutral $W^{0}$ bosons and assume no difference in anomalous interaction for $Z$ and $\gamma$, i.e. $\lambda_{Z}=\lambda_{\gamma}=\lambda$.

Let us consider expression (1.2) as the new free Lagrangian $L_{0}$, whereas expression (1.3) as the new interaction Lagrangian $L_{i n t}$. It is important to note, that we put into the new free Lagrangian the full quadratic in $W$ term including boson self-interaction, because we prefer to maintain gauge invariance of the approximation being used. Indeed, we shall use both quartic term from the last term in (1.2) and triple one from the last but one term of (1.2). Then compensation conditions (see for details [1]) will consist in demand of full connected three-gluon vertices of the structure (1.4), following from Lagrangian $L_{0}$, to be zero. This demand gives a non-linear equation for form-factor $F$.
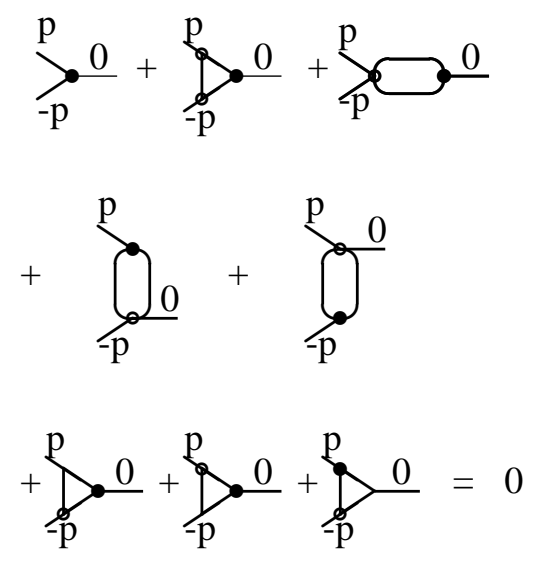

Figure 1: Diagram representation of the compensation equation. Black spot corresponds to anomalous three-gluon vertex with a form-factor. Empty circles correspond to point-like anomalous three-gluon and four-gluon vertices. Simple point corresponds to usual gauge vertex. Incoming momenta are denoted by the corresponding external lines.

Now in view of obtaining the first approximation we would make the following assumptions. 1) In compensation equation we restrict ourselves by terms with loop numbers 0,1 .

2) We reduce thus obtained non-linear compensation equation to a linear integral equation. It means that in loop terms only one vertex contains the form-factor, being defined above, while other vertices are considered to be point-like. In diagram form equation for form-factor $F$ is presented in 1. Here four-leg vertex correspond to interaction of four bosons due to our effective threefield interaction. In our approximation we take here point-like vertex with interaction constant 
proportional to $g G$.

3) We integrate by angular variables of the 4-dimensional Euclidean space.

We look for a solution with the following simple dependence on all three variables

$$
F\left(p_{1}, p_{2}, p_{3}\right)=F\left(\frac{p_{1}^{2}+p_{2}^{2}+p_{3}^{2}}{2}\right) ;
$$

Thus we have

$$
\begin{aligned}
& F(x)=-\frac{G^{2} N}{64 \pi^{2}}\left(\int_{0}^{Y} F(y) y d y-\frac{1}{12 x^{2}} \int_{0}^{x} F(y) y^{3} d y+\frac{1}{6 x} \int_{0}^{x} F(y) y^{2} d y+\right. \\
& \left.+\frac{x}{6} \int_{x}^{Y} F(y) d y-\frac{x^{2}}{12} \int_{x}^{Y} \frac{F(y)}{y} d y\right)+\frac{G g N}{16 \pi^{2}} \int_{0}^{Y} F(y) d y+ \\
& +\frac{G g N}{24 \pi^{2}}\left(\int_{3 x / 4}^{x} \frac{(3 x-4 y)^{2}(2 y-3 x)}{x^{2}(x-2 y)} F(y) d y+\int_{x}^{Y} \frac{(5 x-6 y)}{(x-2 y)} F(y) d y\right)+ \\
& +\frac{G g N}{32 \pi^{2}}\left(\int_{3 x / 4}^{x} \frac{3(4 y-3 x)^{2}\left(x^{2}-4 x y+2 y^{2}\right)}{8 x^{2}(2 y-x)^{2}} F(y) d y+\int_{x}^{Y} \frac{3\left(x^{2}-2 y^{2}\right)}{8(2 y-x)^{2}} F(y) d y+\right. \\
& \left.+\int_{0}^{x} \frac{5 y^{2}-12 x y}{16 x^{2}} F(y) d y+\int_{x}^{Y} \frac{3 x^{2}-4 x y-6 y^{2}}{16 y^{2}} F(y) d y\right)
\end{aligned}
$$

Here $x=p^{2}$ and $y=q^{2}$, where $q$ is an integration momentum, $N=2$. The last four terms in brackets represent diagrams with one usual gauge vertex (see three last diagrams at 1 ). We introduce here an effective cut-off $Y$, which bounds a "low-momentum" region where our non-perturbative effects act and consider the equation at interval $[0, Y]$ under condition

$$
F(Y)=0 .
$$

We shall solve equation (1.8) by iterations. The second iterations gives the following equation

$$
\begin{aligned}
& F(z)=1+\frac{85 g \sqrt{N} \sqrt{z}}{96 \pi}\left(\ln z+4 \gamma+4 \ln 2+\frac{1}{2} G_{15}^{31}\left(\left.z_{0}\right|_{0,0,1 / 2,-1,-1 / 2} ^{0}\right)-\right. \\
& \left.-\frac{595}{336}\right)+\frac{2}{3 z} \int_{0}^{z} F(t) t d t-\frac{4}{3 \sqrt{z}} \int_{0}^{z} F(t) \sqrt{t} d t-\frac{4 \sqrt{z}}{3} \int_{z}^{z_{0}} F(t) \frac{d t}{\sqrt{t}}+ \\
& +\frac{2 z}{3} \int_{z}^{z_{0}} F(t) \frac{d t}{t} ;
\end{aligned}
$$

where $\gamma$ is the Euler constant. We look for solution of (1.10) in the form

$$
\begin{aligned}
& F(z)=\frac{1}{2} G_{15}^{31}\left(\left.z\right|_{1,1 / 2,0,-1 / 2,-1} ^{0}\right)-\frac{85 g \sqrt{N}}{512 \pi} G_{15}^{31}\left(\left.z\right|_{1,1 / 2,1 / 2,-1 / 2,-1} ^{1 / 2}\right)+ \\
& +C_{1} G_{04}^{10}(z \mid 1 / 2,1,-1 / 2,-1)+C_{2} G_{04}^{10}(z \mid 1,1 / 2,-1 / 2,-1) .
\end{aligned}
$$

where

$$
G_{q p}^{n m}\left(\left.z\right|_{b_{1}, \ldots, b_{p}} ^{a_{1}, \ldots, a_{q}}\right)
$$


is a Meijer function. In case $q=0$ we write only indices $b_{i}$ in one line. Constants $C_{1}, C_{2}$ are defined by boundary conditions. We have also conditions

$$
\begin{aligned}
& 1+8 \int_{0}^{z_{0}} F(z) d z=\frac{87 g \sqrt{N}}{32 \pi} \int_{0}^{z_{0}} F_{0}(z) \frac{d z}{\sqrt{z}} ; \\
& F\left(z_{0}\right)=0 .
\end{aligned}
$$

Knowing form (1.11) of a solution we calculate both sides of the equation in two different points in interval $0<z<z_{0}$ and having four equations for four parameters solve the set. With $N=2$ this gives

$$
g\left(z_{0}\right)=0.60366 ; \quad z_{0}=9.61750 ; \quad C_{1}=-0.035096 ; \quad C_{2}=-0.051104 .
$$

We consider the neglected terms of the equation as perturbations to be taken into account in forthcoming studies.

We have one-loop expression for $\alpha_{s}\left(p^{2}\right)$

$$
\alpha_{e w}(x)=\frac{6 \pi \alpha_{e w}\left(x_{0}\right)}{6 \pi+5 \alpha_{e w}\left(x_{0}\right) \ln \left(x / x_{0}\right)} ; \quad x=p^{2} ;
$$

We normalize the running coupling by condition

$$
\alpha_{e w}\left(x_{0}\right)=\frac{g(Y)^{2}}{4 \pi}=0.0290 ;
$$

Note that value (1.16) is not far from physical value $\alpha_{e w}\left(M_{W}\right)=0.0337$. To compare these values properly one needs a relation connecting $G$ and $M_{W}$. For example with $|g \lambda|=0.025, \alpha_{e w}\left(M_{W}\right)=$ 0.0312 . The experimental value 0.0337 is reached for $|g \lambda|=0.000003$. For both cases values of $\lambda$ are consistent with limitations (1.6). Bearing in mind that accuracy of the present approximation is estimted to be $\simeq 10 \%$ we can state that agreement is valid for all possible values of $\lambda$. In what follows we shall use experimental value $\alpha_{e w}\left(M_{W}\right)=0.0337$.

\section{Four-fermion interaction of heavy quarks}

Let us remind that the adequate description of low-momenta region in QCD can be achieved by an introduction of the effective Nambu - Jona-Lasinio interaction [15, 16] (see recent review [17]). In the framework of the compensation approach the spontaneouis generation of NJL-type interaction was demonstrated in works [2, 3]. In these works pions are described as bound states of light quarks, which are formed due to the effective NJL interaction with account of QCD corrections.

In the present work we explore the analogous considerations and assume that scalar fields which substitute elementary Higgs fields are formed by bound states of heavy quarks $t, b$. This possibility was proposed (1989 - 1990) in works by Y.Nambu, V.Miransky, M.Tanabashi, K.Yamawaki, W.Bardeen, C.Hill, M.Lindner [18, 19, 20] and was considered in a number of publications (see, e.g. review by M.Lindner [21]). It comes clear, that estimates of mass of the $t$-quark in this model gives result which exceeds significantly its measured value. In the present work we obtain the fourfermion interaction in the framework of Bogoliubov compensation approach, while in the previous 
works on the model the interaction was postulated. In our approach parameters of the problem are obtained as an unique solution of a set of equations quite analogously [2, 3]. In particular we shall see that the $t$-quark mass is quite consistent with the current data.

We have started with Lagrangian (1.1) in which both gauge bosons $W$ and spinor particles (leptons and quarks) are massless. As the first stage we consider approximation in which only the most heavy particles aquire masses, namely $W$-s and the $t$-quark while all other ones remain massless. In view of this we introduce left doublet $\Psi_{L}=\left(1+\gamma_{5}\right) / 2 \cdot(t, b)$ and right singlet $T_{R}=$ $\left(1-\gamma_{5}\right) / 2 \cdot t$. Then we study a possibility of spontaneous generation [1, 2, 3, 5] of the following effective non-local four-fermion interaction

$$
\begin{aligned}
& L_{f f}=G_{1} \bar{\Psi}_{L}^{\alpha} T_{R \alpha} \bar{T}_{R}^{\beta} \Psi_{L \beta}+G_{2} \bar{\Psi}_{L}^{\alpha} T_{R \beta} \bar{T}_{R}^{\beta} \Psi_{L \alpha}+ \\
& \frac{G_{3}}{2} \bar{\Psi}_{L}^{\alpha} \gamma_{\mu} \Psi_{L \alpha} \bar{\Psi}_{L}^{\beta} \gamma_{\mu} \Psi_{L \beta}+\frac{G_{4}}{2} \bar{T}_{R}^{\alpha} \gamma_{\mu} T_{R \alpha} \bar{T}_{R}^{\beta} \gamma_{\mu} T_{R \beta} .
\end{aligned}
$$

where $\alpha, \beta$ are colour indices. We shall formulate and solve compensation equations for formfactors of the first two interaction, while consideration of the two last ones is postponed for the next approximations. Here we follow the procedure used in works, which deal with four-fermion Nambu-Jona-Lasinio interaction. However coupling constants $G_{3}, G_{4}$ essentially influence the forthcoming results. In this section $N=3$ and a kernel term in equations is the following

$$
\begin{aligned}
& K \times F=\left(\Lambda^{2}-x \ln \Lambda^{2}\right) \int_{0}^{\bar{Y}} F(y) d y-\ln \Lambda^{2} \int_{0}^{\bar{Y}} F(y) y d y+ \\
& \frac{1}{6 x} \int_{0}^{x} F(y) y^{2} d y+\ln x \int_{0}^{x} F(y) y d y+x\left(\ln x-\frac{3}{2}\right) \int_{0}^{x} F(y) d y+ \\
& \int_{x}^{\bar{Y}} y\left(\ln y-\frac{3}{2}\right) F(y) d y+x \int_{x}^{\bar{Y}} \ln y F(y) d y+\frac{x^{2}}{6} \int_{x}^{\bar{Y}} \frac{F(y)}{y} d y .
\end{aligned}
$$

$\Lambda$ is auxiliary cut-off, which disappears from all expressions with all conditions for solutions be fulfilled. The compensation equation corresponds to set of diagrams at 2

$$
\begin{gathered}
\Phi(x)=\frac{\Lambda^{2}\left(N^{2} G_{1}^{2}+2 N G_{1} G_{2}+G_{2}^{2}\right)}{8 \pi^{2}\left(N G_{1}+G_{2}\right)}\left(1-\frac{N G_{1}+G_{2}}{8 \pi^{2}} \int_{0}^{\bar{Y}} \Phi(y) d y\right)+ \\
\left(\Lambda^{2}+\frac{x}{2} \ln \frac{x}{\Lambda^{2}}-\frac{3 x}{4}\right) \frac{G_{1}^{2}+G_{2}^{2}+2 N G_{1} G_{2}+2 \bar{G}(N+1)\left(G_{1}+G_{2}\right)}{32 \pi^{2}\left(N G_{1}+G_{2}\right)}- \\
\frac{G_{1}^{2}+G_{2}^{2}+2 N G_{1} G_{2}+2 \bar{G}(N+1)\left(G_{1}+G_{2}\right)}{2^{9} \pi^{4}} K \times \Phi ; \\
F_{2}(x)=\frac{\Lambda^{2} G_{2}}{8 \pi^{2}}\left(1-\frac{G_{2}}{8 \pi^{2}} \int_{0}^{\bar{Y}} F_{2}(y) d y\right)+ \\
\left(\Lambda^{2}+\frac{x}{2} \ln \frac{x}{\Lambda^{2}}-\frac{3 x}{4}\right) \frac{G_{1}^{2}+G_{2}^{2}+2 \bar{G}\left(G_{1}+G_{2}(N+1)\right)}{32 \pi^{2} G_{2}}- \\
\frac{G_{1}^{2}+G_{2}^{2}+2 \bar{G}\left(G_{1}+G_{2}(N+1)\right.}{2^{9} \pi^{4}} K \times F_{2} ; \Phi(\bar{Y})=F_{2}(\bar{Y})=0 ; \\
\Phi(x)=\frac{N G_{1} F_{1}+G_{2} F_{2}}{N G_{1}+G_{2}} ; \quad \bar{G}=\frac{G_{3}+G_{4}}{2} ; \quad x=p^{2} ; \quad y=q^{2} .
\end{gathered}
$$




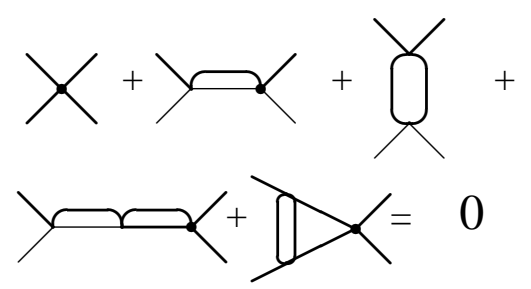

Figure 2: Diagram representation of the compensation equation for the four-fermion interaction. Lines describe quarks. Simple point corresponds to the point-like vertex and black circle corresponds to a vertex with a form-factor.

Introducing substitution $G_{1}=\rho \bar{G}, G_{2}=\omega \bar{G}$ and comparing the two equations $(2.3,2.4)$ we get convinced, that both equations become being the same under the following condition

$$
\rho=0 \text {. }
$$

and we are rested with one equation

$$
\begin{aligned}
& F_{2}(z)=\frac{\sqrt{\omega^{2}+8 \omega}}{\omega} \sqrt{z}(\ln z-3)-16\left[\frac{1}{6 \sqrt{z}} \int_{0}^{z} F_{2}(t) \sqrt{t} d t+\right. \\
& \frac{\ln z}{2} \int_{0}^{z} F_{2}(t) d t+\frac{\sqrt{z}(\ln z-3)}{2} \int_{0}^{z} \frac{F_{2}(t)}{\sqrt{t}} d t+ \\
& \left.\frac{1}{2} \int_{z}^{\bar{z}_{0}}(\ln t-3) F_{2}(t) d t+\frac{\sqrt{z}}{2} \int_{z}^{\bar{z}_{0}} \ln t \frac{F_{2}(t)}{\sqrt{t}} d t+\frac{z}{6} \int_{z}^{\bar{z}_{0}} \frac{F_{2}(t)}{t} d t\right] ; \\
& z=\frac{\left(\omega^{2}+8 \omega\right) \bar{G}^{2} x^{2}}{2^{14} \pi^{4}} ; \quad t=\frac{\left(\omega^{2}+8 \omega\right) \bar{G}^{2} y^{2}}{2^{14} \pi^{4}} ; \quad \bar{z}_{0}=\frac{\left(\omega^{2}+8 \omega\right) \bar{G}^{2} \bar{Y}^{2}}{2^{14} \pi^{4}} .
\end{aligned}
$$

Here we omit all terms containing auxiliary cut-off $\Lambda$ due to their cancellation.

Performing consecutive differentiations of Eq.(2.6) we obtain the following differential equation for $F_{2}$

$$
\begin{aligned}
& \left(z \frac{d}{d z}+\frac{1}{2}\right)\left(z \frac{d}{d z}\right)\left(z \frac{d}{d z}\right)\left(z \frac{d}{d z}-\frac{1}{2}\right)\left(z \frac{d}{d z}-\frac{1}{2}\right) \times \\
& \left(z \frac{d}{d z}-1\right) F_{2}(z)+z F_{2}(z)=0
\end{aligned}
$$

The equation is equivalent to integral equation (2.6) provided the following boundary conditions being fulfilled

$$
\begin{aligned}
& \int_{0}^{\bar{z}_{0}} \frac{F_{2}(t)}{\sqrt{t}} d t=\frac{\sqrt{\omega^{2}+8 \omega}}{8 \omega} ; \quad F_{2}\left(\bar{z}_{0}\right)=0 ; \\
& \int_{0}^{\bar{z}_{0}} F_{2}(t) \sqrt{t} d t=0 ; \quad \int_{0}^{\bar{z}_{0}} F_{2}(t) d t=0 .
\end{aligned}
$$

Note that just boundary conditions (2.8) lead to cancellation of all terms containing $\Lambda$. Differential equation is a Meijer equation and the solution of the problem is the following

$$
F_{2}(z)=\frac{1}{2 \sqrt{\pi}} G_{06}^{40}\left(z \mid 0, \frac{1}{2}, \frac{1}{2}, 1,-\frac{1}{2}, 0\right) ; \quad z_{0}=\infty .
$$




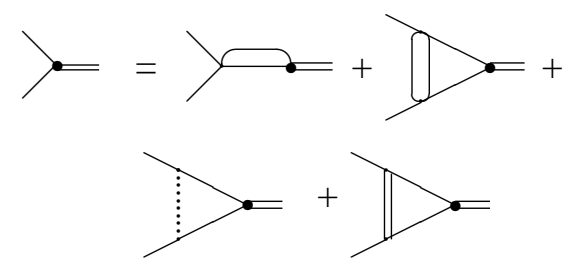

Figure 3: Diagram representation of the Bethe-Salpeter equation for a bound state of heavy quarks. Double line represent the bound state and dotted line describes a gluon. Black circle corresponds to BS wave function. Other notations are the same as at Fig. 2.

Here we also take into account condition $F_{2}(0)=1$ that gives $\omega=\frac{8}{3}$.

We would draw attention to the fact, that unique solution (2.9) exists only for infinite upper limit in integrals.

\section{Doublet bound state $\bar{\Psi}_{L} T_{R}$}

Let us study a possibility of spin-zero doublet bound state $\bar{\Psi}_{L} T_{R}=\phi$, which can be referred to a Higgs scalar. With account of interaction (2.1) using results of the previous section we have the following Bethe-Salpeter equation, in which we take into account the $t$-quark mass (see 3)

$$
\Psi(x)=\frac{\bar{G}}{16 \pi^{2}} \int \Psi(y) d y+\frac{G_{2}^{2}}{2^{7} \pi^{4}} K^{*} \times \Psi ;
$$

where the modified integral operator $K^{*}$ is defined in the same way as operator (2.2) with $\bar{Y}=\infty$ and lower limit of integration 0 being changed for the $t$-quark mass $m_{t}^{2}$. Then we have again differential equation

$$
\begin{aligned}
& \left(z \frac{d}{d z}-a_{1}\right)\left(z \frac{d}{d z}-a_{2}\right)\left(z \frac{d}{d z}\right)\left(z \frac{d}{d z}-\frac{1}{2}\right)\left(z \frac{d}{d z}-\frac{1}{2}\right)\left(z \frac{d}{d z}-1\right) \Psi(z)-z \Psi(z)=0 ; \\
& a_{1}=-\frac{1+\sqrt{1+64 \mu}}{4} ; \quad a_{2}=-\frac{1-\sqrt{1+64 \mu}}{4} ; \quad \mu=\frac{G_{2}^{2} m^{4}}{2^{12} \pi^{4}} ;
\end{aligned}
$$

where the main difference is the other sign of the last term, while variable $z$ is just the same as in (2.6) with account of relation $\omega=\frac{8}{3}$. Boundary conditions now are the following

$$
\int_{\mu}^{\infty} \frac{\Psi(t)}{\sqrt{t}} d t=0 ; \int_{\mu}^{\infty} \Psi(t) \sqrt{t} d t=0 ; \int_{\mu}^{\infty} \Psi(t) d t=0 ; \Psi(\mu)=1 .
$$

Solution of the problem is presented in the following form

$$
\begin{aligned}
& \Psi(z)=C_{1} G_{06}^{50}\left(z \mid a_{1}, a_{2}, 1 / 2,1 / 2,1,0\right)+C_{2} G_{06}^{30}\left(z \mid 0,1 / 2,1, a_{1}, a_{2}, 1 / 2\right)+ \\
& C_{3} G_{06}^{30}\left(z \mid 1 / 2,1 / 2,1, a_{1}, a_{2}, 0\right)+C_{4} G_{06}^{50}\left(z \mid a_{1}, a_{2}, 0,1 / 2,1,1 / 2\right) ;
\end{aligned}
$$

where $C_{i}$ for given $\mu$ are uniquely defined by boundary conditions. We define interaction of the doublet $\phi$ with heavy quarks

$$
L_{\phi}=g_{\phi}\left(\phi^{*} \bar{\Psi}_{L} T_{R}+\phi \bar{T}_{R} \Psi_{L}\right)
$$




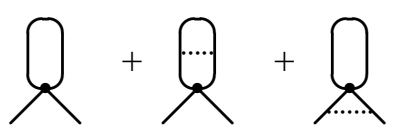

Figure 4: Diagram representation of additional contribution to the $t$-quark mass. Dotted lines represent gluons. Other notations the same as at Fig. 2.

where $g_{\phi}$ is the coupling constant of the new interaction to be defined by normalization condition of the solution of equation (4.6).

Using standard perturbative method we obtain for the mass of the bound state under consideration the following expression in the same way as in [3].

$$
\begin{aligned}
& m_{\phi}^{2}=-\frac{m_{t}^{2} I_{5}}{\sqrt{\pi \mu} I_{2}} ; \quad I_{2}=\int_{\mu}^{\infty} \frac{\Psi(z)^{2} d z}{z} \\
& I_{5}=\int_{\mu}^{\infty} \frac{\left(16 \pi \alpha_{s}(z)-g_{\phi}^{2}\right) \Psi(z) d z}{16 \pi z} \int_{\mu}^{z} \frac{\Psi(t) d t}{\sqrt{t}} .
\end{aligned}
$$

Here $\alpha_{s}(z)$ is the strong coupling with standard evolution, normalized at the $t$-quark mass, and we put $m=m_{t}$. Provided term with brackets inside $I_{5}$ being positive, bound state $\phi$ is a tachyon. Let us recall the well-known relation for $t$-quark mass, which is defined by non-zero vacuum average of $\left(\phi_{2}^{*}+\phi_{2}\right) / \sqrt{2}$. It reads

$$
m_{t}=\frac{g_{\phi} \eta}{\sqrt{2}}
$$

where $\eta=246.2 \mathrm{GeV}$ is the value of the electro-weak scalar condensate.

However in our approach there are additional contribution to this mass, e.g. due to diagram shown at 4

That means that for experimental value of the $t$-quark we take the modified definition

$$
m_{t}=\frac{g_{\phi} \eta}{\sqrt{2}}+\Delta M=\frac{g_{\phi} \eta}{f \sqrt{2}}
$$

According to these diagrams we have the following expression for $\Delta M$

$$
\begin{aligned}
& \Delta M=-4 m_{t} \int_{\mu}^{\infty} \frac{F_{2}(z) d z}{\sqrt{z}} \int_{\mu}^{\infty} \frac{\alpha_{s}(z) F_{2}(z) d z}{2 \pi z}-4 \int_{\mu}^{\infty} \frac{m_{t}(z) F_{2}(z) d z}{\sqrt{z}} \\
& m_{t}(z)=m_{t}\left(1+\frac{7 \alpha_{s}(\mu)}{8 \pi} \ln \frac{z}{\mu}\right)^{-\frac{4}{7}}
\end{aligned}
$$

Here the first term corresponds to gluon exchange between external legs and the second term corresponds to gluon exchanges inside the loop calculated with account of standard RG mass evolution. Contributions of gluon exchanges from external legs to internal lines cancel. Now parameter $f$ defined in (3.7) is the following

$$
f=1+4 \int_{\mu}^{\infty} \frac{F_{2}(z) d z}{\sqrt{z}} \int_{\mu}^{\infty} \frac{\alpha_{s}(z) F_{2}(z) d z}{2 \pi z}+4 \int_{\mu}^{\infty} \frac{m_{t}(z) F_{2}(z) d z}{m_{t} \sqrt{z}} .
$$




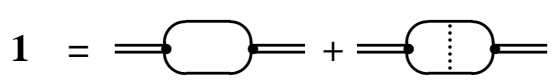

Figure 5: Diagrams for normalization condition for $H \bar{\Psi}_{L} t_{R}$-vertex. Notations are the same as at Figs. 2 - 4.

Due to relation (2.8) factor $f$ in $(3.7,3.9)$ is slightly larger than 2. For strong coupling $\alpha_{s}(z)$ we use the standard one-loop expression

$$
\alpha_{s}(z)=\alpha_{s}(\mu)\left(1+\frac{7 \alpha_{s}(\mu)}{8 \pi} \ln \frac{z}{\mu}\right)^{-1} \cdot \alpha_{s}(\mu)=0.108
$$

where for strong coupling at the $t$-quark mass we take its value obtained by evolution expression (3.10) from its value at $M_{Z}: \alpha_{s}\left(M_{Z}\right)=0.1184 \pm 0.0007$.

Let us consider the possibility when relation (3.5) leads to a tachyon state. For Higgs mechanism to be realized we need also four-fold interaction

$$
\mathrm{Ł}_{\phi 4}=\lambda\left(\phi^{*} \phi\right)^{2} .
$$

Coupling constant in (3.11) is defined in terms of the following loop integral

$$
\lambda=\frac{3 g_{\phi}^{4}}{16 \pi^{2}} I_{4} ; \quad I_{4}=\int_{\mu}^{\infty} \frac{\Psi(z)^{4} d z}{z} .
$$

From well-known relations $\eta^{2}=-m_{\phi}^{2} / \lambda$ and the Higgs mass squared $M_{H}^{2}=-2 m_{\phi}^{2}$ we have

$$
\eta^{2}=\frac{16 \pi m_{t}^{2} I_{5}}{3 g_{\phi}^{4} \sqrt{\mu} I_{2} I_{4}} ; \quad M_{H}^{2}=\frac{2 m_{t}^{2} I_{5}}{\pi \sqrt{\mu} I_{2}} .
$$

From (3.7) and (3.13) we have useful relation

$$
2=\frac{16 \pi I_{5}}{3 g_{\phi}^{2} f^{2} \sqrt{\mu} I_{2} I_{4}} .
$$

We obtain $g_{\phi}$ from a normalization condition, which is defined by diagrams of 5

$$
\begin{aligned}
& \frac{3 g_{\phi}^{2}}{32 \pi^{2}}\left(I_{2}+\frac{\alpha_{s}(\mu)}{4 \pi}\left(I_{22}^{2}+2 I_{6}\right)\right)=1 \\
& I_{22}=\int_{\mu}^{\infty} \frac{\Psi(t) d t}{t} ; \quad I_{6}=\int_{\mu}^{\infty} \frac{\Psi(z) d z}{z \sqrt{z}} \int_{\mu}^{z} \frac{\Psi(t) d t}{\sqrt{t}} .
\end{aligned}
$$

Here we use strong coupling at the $t$-quark mass (3.10) and perform necessary calculations. In doing this we proceed in the following way: for six parameters $\mu, g_{\phi}, \eta, m_{t}, M_{H}, f$ we have five relations (3.7, 3.13, 3.14, 3.16) and the well-known expression

$$
M_{W}=\frac{g_{w} \eta}{2}
$$


where $g_{w}$ is weak interaction constant $g$ at $W$ mass. We obtain it by usual RG evolution expression (1.15) from value $g$ at $Y$ (1.14). Let us remind that we consider $M_{W}$ as an input. Thus for the moment we have two input parameters, which are safely known from the experiment

$$
M_{W}=80.4 \mathrm{GeV} ; \quad \eta=246.2 \mathrm{GeV} .
$$

The last value corresponds to value of electro-weak coupling $g_{w}\left(M_{W}\right)=0.653$.

Now we present thus obtained parameters

$$
\begin{aligned}
& \mu=4.067510^{-12} ; \quad f=2.034 ; \quad g_{\phi}=2.074 ; \\
& m_{t}=177.0 \mathrm{GeV} ; \quad M_{H}=1803 \mathrm{GeV} .
\end{aligned}
$$

The most important result here is the $t$-quark mass, which is close to experimental value $M_{t}=$ $173.3 \pm 1.1 \mathrm{GeV}$ [23]. Really, the main difficulty of composite Higgs models [18, 19, 20, 21] consists in too large $m_{t}$. Indeed the definition of $g_{\phi}$ in such models leads to $g_{\phi} \simeq 3$ and thus $m_{t} \simeq 500 \mathrm{GeV}$. In the present work we have all parameters, including inportant parameter $f$, being defined by selfconsistent set of equations and the unique solution gives results (3.18), which for $m_{t}$ is quite satisfactory. The large value for $M_{H}$ seems to contradict to upper limit for this mass, which follows from considerations of Landau pole in the $\lambda \phi^{4}$ theory. Emphasize, that this limit corresponds to the local theory and in our case of composite scalar fields is not relevant. Such large mass of $H$ means, of course, very large width of $H$

$$
\begin{aligned}
& \Gamma_{H}=3784 \mathrm{GeV} ; \quad B R\left(H \rightarrow W^{+} W^{-}\right)=51.4 \% \\
& B R(H \rightarrow Z Z)=25.6 \% ; \quad B R(H \rightarrow \bar{t} t)=23.0 \% .
\end{aligned}
$$

Thus our approach predicts, that unfortunately quest for Higgs particle at LHC will give negative result. Maybe one could succeed in registration of slight increasing of cross-sections $p+p \rightarrow$ $W^{+}+W^{-}+X, p+p \rightarrow Z+Z+X, p+p \rightarrow \bar{t}+t+X$ in region of invariant masses of two heavy particles $1 \mathrm{TeV}<M_{12}<3 \mathrm{TeV}$. As a matter of fact the most recent LHC data (SMS PAS-HIG-11022, ATLAS-CONF-2011-135) do not find the SM Higgs in wide interval up to $466 \mathrm{GeV}$ (see also recent ATLAS result [22]).

\section{W-hadrons and CDF Wjj anomaly}

Thus we have triple interaction

$$
\begin{aligned}
& -\frac{G}{3 !} \cdot \varepsilon_{a b c} W_{\mu \nu}^{a} W_{v \rho}^{b} W_{\rho \mu}^{c} ; \\
& W_{\mu \nu}^{3}=\cos \theta_{W} Z_{\mu \nu}+\sin \theta_{W} A_{\mu \nu} ;
\end{aligned}
$$

with uniquely defined form-factor $F\left(p_{i}\right)$. It was done of course in the framework of an approximate scheme, which accuracy was estimated to be $\simeq(10-15) \%$.

Interaction (4.1) increases with increasing momenta $p$ and corresponds to effective dimensionless coupling being of the following order of magnitude

$$
g_{e f f}=\frac{g \lambda p^{2}}{M_{W}^{2}}
$$




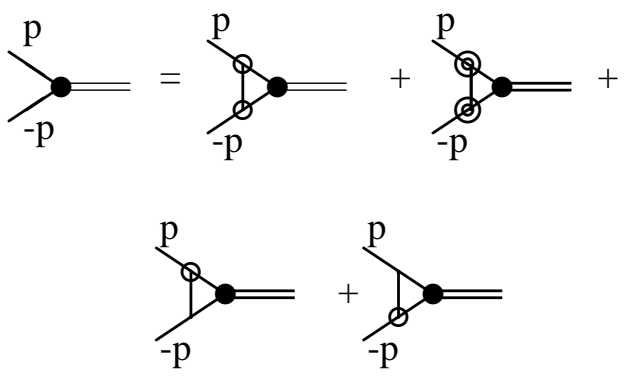

Figure 6: Diagram representation of Bethe-Salpeter equation for W-W bound state. Black spot corresponds to BS wave function. Empty circles correspond to point-like anomalous three-gluon vertex, double circle XWW vertex. Simple point - usual gauge triple $W$ interaction. Double line - the bound state $X$, simple line - W. Incoming momenta are denoted by the corresponding external lines.

Thus for sufficiently large momentum interaction (4.1) becomes strong and may lead to physical consequences analogous to that of the usual strong interaction (QCD). In particular bound states and resonances constituting of $W$-s (W-hadrons) may appear. Let us estimate the typical scale for the effect. We know that in QCD upper bound of a region of really strong interaction (nonperturbative region) is around $600 \mathrm{MeV}$ where $\alpha_{s} \simeq 0.5$ that is coupling $g_{s}=\sqrt{4 \pi \alpha_{s}}=2.5$. So we have to equate $g_{\text {eff }}(4.2)$ to this value and define the typical value $p_{t y p}$ that gives

$$
p_{t y p}=M_{W} \sqrt{\frac{g_{e f f}}{g \lambda}} \simeq 650 \mathrm{GeV}
$$

where we have taken for modulus of $\lambda$ its maximal value from limitation (1.6). Now we have the lightest hadron - the pion with mass $\simeq 140 \mathrm{MeV}$ for typical scale $600 \mathrm{MeV}$ in QCD and for estimated $p_{t y p}$ (4.3) we have possible mass of the lightest W-hadron

$$
M_{\min }=\frac{p_{t y p} M_{\pi}}{600 \mathrm{MeV}} \simeq 150 \mathrm{GeV}
$$

The excess detected in work [27] is situated just in this region. So one might try to consider interpretation of effect [27] as a manifestation of a $W$-hadron.

Here we would apply these considerations along with previous results to data indicating on an excess in jet pair production accompanied by $W$ at TEVATRON [27] in region of $j j$ invariant mass $120-160 \mathrm{GeV}$.

Let us assume that this excess is due to existence of bound state $X$ of two $W$. This state $X$ is assumed to have spin 1 and weak isotopic spin also 1 . Then vertex of $X W W$ interaction has the following form

$$
\frac{G_{X}}{2} \varepsilon_{a b c} W_{\mu \nu}^{a} W_{v \rho}^{b} X_{\rho \mu}^{c} \Psi
$$

where $\Psi$ is a Bethe-Salpeter wave function of the bound state. The main interactions forming the bound state are just non-perturbative interactions $(4.1,4.5)$. This means that we take into account exchange of vector boson $W$ as well as of vector bound state $X$ itself. In diagram form the corresponding Bethe-Salpeter equation is presented in 6 . 
For small mass $M_{X}$ of state $X$ we expand the kernel of the equation in powers of $M_{W}^{2}$ and $M_{X}^{2}$ and obtain the following equation

$$
\begin{aligned}
& \Psi(x)=\frac{G^{2}+G_{X}^{2}}{32 \pi^{2}} \int_{0}^{Y_{0}} \Psi(y) y d y-\frac{G^{2}+G_{X}^{2}}{32 \pi^{2}}\left(\frac{1}{12 x^{2}} \int_{0}^{x} \Psi(y) y^{3} d y-\right. \\
& \left.\frac{1}{6 x} \int_{0}^{x} \Psi(y) y^{2} d y-\frac{x}{6} \int_{x}^{Y_{0}} \Psi(y) d y+\frac{x^{2}}{12} \int_{x}^{Y_{0}} \frac{\Psi(y)}{y} d y\right)+\frac{g G}{4 \pi^{2}}\left(\int_{0}^{Y_{0}} \Psi(y) d y-\right. \\
& \frac{3}{8 x^{3}} \int_{0}^{x} \Psi(y) y^{3} d y+\frac{7}{8 x^{2}} \int_{0}^{x} \Psi(y) y^{2} d y-\frac{1}{2 x} \int_{0}^{x} \Psi(y) y d y+ \\
& \left.\left.\frac{x}{8} \int_{x}^{Y_{0}} \frac{\Psi(y)}{y} d y-\frac{x^{2}}{8} \int_{x}^{Y_{0}} \frac{\Psi(y)}{y^{2}} d y\right)\right)-\frac{\mu \bar{G} \sqrt{2}}{\pi}\left(\int_{0}^{Y_{0}} \Psi(y) d y-\right. \\
& \frac{1}{12 x^{2}} \int_{0}^{x} \Psi(y) y^{2} d y+\frac{1}{6 x} \int_{0}^{x} \Psi(y) y d y+\frac{x}{6} \int_{x}^{Y_{0}} \frac{\Psi(y)}{y} d y \\
& \left.-\frac{x^{2}}{12} \int_{x}^{Y_{0}} \frac{\Psi(y)}{y^{2}} d y\right)-\frac{\chi \bar{G} \sqrt{2}}{\pi}\left(\frac{1}{24} \int_{0}^{Y_{0}} \Psi(y) d y-\frac{1}{192 x^{3}} \int_{0}^{x} \Psi(y) y^{3} d y+\right. \\
& \left.\frac{1}{64 x} \int_{0}^{x} \Psi(y) y d y+\frac{x}{64} \int_{x}^{Y_{0}} \frac{\Psi(y)}{y}-\frac{x^{3}}{192} \int_{x}^{Y_{0}} \frac{\Psi(y)}{y^{3}} d y\right) . \\
& \mu=\frac{\bar{G} M_{W}^{2}}{16 \pi \sqrt{2}} ; \chi=\frac{\bar{G} M_{X}^{2}}{16 \pi \sqrt{2}} ; \bar{G}=\sqrt{G^{2}+G_{X}^{2}} .
\end{aligned}
$$

Here $x=p^{2}$ is the external momentum squared and $y$ is the integration momentum squared. Gauge electro-weak coupling $g$ enters due to diagrams of the second line of 6 Upper limit $Y_{0}$ is introduced for the sake of generality due the experience of works [2, 3, 4, 6, 24], according to which $Y_{0}$ may be either $\infty$ or some finite quantity. That is $Y_{0}$ is defined in a process of solving an equation. From the physical point of view an effective cut-off $Y_{0}$ bounds a "low-momentum" region where our non-perturbative effects act and we consider the equation at interval $\left[0, Y_{0}\right]$ under condition

$$
\Psi\left(Y_{0}\right)=0 .
$$

For interaction (4.1) $Y_{0}$ is already defined.

We shall solve equation (4.6) by iterations. Let us perform the following substitution

$$
z=\frac{\left(G^{2}+G_{X}^{2}\right) x^{2}}{512 \pi^{2}}, \quad t=\frac{\left(G^{2}+G_{X}^{2}\right) y^{2}}{512 \pi^{2}}
$$

then the zero approximation reads

$$
\Psi_{00}(z)=\frac{\pi}{2} G_{15}^{21}\left(\left.z\right|_{1,0,1 / 2,-1 / 2,-1} ^{0}\right) .
$$

Now set of equations (4.6) takes the form

$$
\begin{aligned}
& \Psi_{0}(z)=I N H-\frac{2}{3 z} \int_{0}^{z} \Psi_{0}(t) t d t+\frac{4}{3 \sqrt{z}} \int_{0}^{z} \Psi_{0}(t) \sqrt{t} d t+ \\
& \frac{4 \sqrt{z}}{3} \int_{z}^{z_{0}^{\prime}} \frac{\Psi_{0}(t)}{\sqrt{t}} d t-\frac{2 z}{3} \int_{z}^{z_{0}^{\prime}} \frac{\Psi_{0}(y)}{y} d y
\end{aligned}
$$




$$
\begin{aligned}
& I N H=1-\sqrt{z}\left(\frac{g^{\prime} \sqrt{2}}{8 \pi}+\frac{8 \mu}{3}-\frac{\chi}{4}\right)(\ln z+4 \gamma+4 \ln 2+ \\
& \left.\frac{\pi}{2} G_{15}^{21}\left(\left.z_{0}^{\prime}\right|_{0,0,1 / 2,-1 / 2,-1} ^{0}\right)\right)+\sqrt{z}\left(\frac{g^{\prime} \sqrt{2}}{48 \pi}+\frac{68 \mu}{9}-\frac{25 \chi}{32}\right) \\
& 1=8 \int_{0}^{z_{0}^{\prime}} \Psi_{0}(t) d t-\left(\frac{g^{\prime} 2 \sqrt{2}}{\pi}-16 \mu+\frac{2 \chi}{3}\right) \int_{0}^{z_{0}^{\prime}} \frac{\Psi_{00}(t)}{\sqrt{t}} d t
\end{aligned}
$$

where $\gamma$ is the Euler constant. Now we look for solutions of set (4.10). We have relation

$$
M_{X}=M_{W} \sqrt{\frac{\chi}{\mu}} ; \quad M_{W}=80.4 \mathrm{GeV} .
$$

We look for the exact solution of set of equations (4.10) in the following form

$$
\begin{aligned}
& \Psi_{0}(z)=\frac{\pi}{2} G_{15}^{21}\left(\left.z\right|_{1,0,1 / 2,-1 / 2,-1} ^{0}\right)+ \\
& C_{1} G_{15}^{21}\left(\left.z\right|_{1 / 2,1 / 2,1,-1 / 2,-1} ^{1 / 2}\right)+ \\
& C_{2} G_{04}^{20}\left(\left.z\right|_{1,1 / 2,-1 / 2,-1}\right)+ \\
& C_{3} G_{04}^{10}\left(-\left.z\right|_{1,1 / 2,-1 / 2,-1}\right) .
\end{aligned}
$$

Let us choose a solution with "experimental" $M_{X}=145 \mathrm{GeV}$ [27], then we have solution with the following parameters

$$
\begin{aligned}
& C_{1}=-0.015282 ; \quad C_{2}=-3.26512 ; \\
& C_{3}=1.2796210^{-11} ; \quad g^{\prime}=0.03932 ; \\
& \chi=0.0074995 ; \quad z_{0}^{\prime}=2627.975 ; \\
& \mu=0.001824 .
\end{aligned}
$$

Parameters (4.13) defines the following physical parameters

$$
\begin{aligned}
& G=\frac{0.0099}{M_{W}^{2}} ; \lambda=-\frac{G M_{W}^{2}}{g}=-0.0152 ; \\
& M_{X}=145 \mathrm{GeV} ; \quad\left|G_{X}\right|=\frac{0.1639}{M_{W}^{2}} .
\end{aligned}
$$

Value $\lambda$ (4.14) agrees with restrictions (1.6). Note, that set (4.10) with $M_{X}=145 \mathrm{GeV}$ has also few other solutions, but they lead to values of $\lambda$ which contradict to restrictions (1.6). On the other hand with value of $G$ from (4.14) we have additional solutions for "radial excitation" $X$ with mass and coupling constant

$$
\begin{array}{ll}
M_{X_{1}}=180.7 \mathrm{GeV} ; & \left|G_{X}\right|=\frac{0.0628}{M_{W}^{2}} ; \\
M_{X_{2}}=205.1 \mathrm{GeV} ; & \left|G_{X}\right|=\frac{0.1155}{M_{W}^{2}} ; \\
M_{X_{3}}=244.2 \mathrm{GeV} ; & \left|G_{X}\right|=\frac{0.1837}{M_{W}^{2}} .
\end{array}
$$




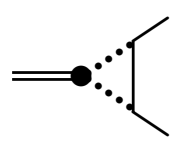

Figure 7: Diagram for vertex $X \bar{q} q$. Dotted line - W, double line - bound state $X$, simple line - a quark. Black spot - the $X W W$ BS wave function.

With this mass $X$ decay into pair of $W$-s, i.e.

$$
\begin{aligned}
& X_{1,2,3}^{ \pm} \rightarrow W^{ \pm}+(Z, \gamma) ; \quad X_{1,2,3}^{0} \rightarrow W^{+}+W^{-} ; \\
& \Gamma\left(X_{1}^{0}\right)=0.0086 \mathrm{GeV} ; \quad \Gamma\left(X_{1}^{ \pm}\right)=0.0051 \mathrm{GeV} ; \\
& \Gamma\left(X_{2}^{0}\right)=0.126 \mathrm{GeV} ; \quad \Gamma\left(X_{2}^{ \pm}\right)=0.083 \mathrm{GeV} ; \\
& \Gamma\left(X_{3}^{0}\right)=1.26 \mathrm{GeV} ; \quad \Gamma\left(X_{3}^{ \pm}\right)=0.89 \mathrm{GeV} ; \\
& B R\left(X_{1}^{+} \rightarrow W Z=0.44 ; \quad B R\left(X_{1}^{+} \rightarrow W \gamma\right)=0.56 ;\right. \\
& B R\left(X_{2}^{+} \rightarrow W Z=0.80 ; \quad B R\left(X_{2}^{+} \rightarrow W \gamma\right)=0.20 ;\right. \\
& B R\left(X_{3}^{+} \rightarrow W Z=0.91 ; \quad B R\left(X_{3}^{+} \rightarrow W \gamma\right)=0.09 .\right.
\end{aligned}
$$

Now interaction (4.5) with parameters (4.14) defines reactions of $X^{ \pm}, X^{0}$ production at TEVATRON and their decays. Bound states $X$ interact with fermion doublets $\psi_{L}$ due to diagram presented at 7. The effective interaction is described by the following expression

$$
\begin{aligned}
& L_{X \psi}=g_{X} X_{v}^{a} \bar{\psi}_{L} \tau^{a} \gamma^{v} \psi_{L} \\
& g_{X}=\frac{g^{2} G_{X} M_{X}^{2}}{64 \pi^{2}} \int_{\mu^{2}}^{z_{0}} \frac{\Psi_{0}(t)}{t} d t=0.0006704
\end{aligned}
$$

Due to interactions $(4.5,4.17)$ there are the following decays of bound states $X$

$$
\begin{aligned}
& X^{ \pm} \rightarrow W^{ \pm}+\gamma ;(85.9 \%) ; \quad X^{ \pm} \rightarrow u \bar{d}(d \bar{u})(9.5 \%) ; \\
& X^{0} \rightarrow u \bar{u} ;(71.4 \%) ;
\end{aligned}
$$

Taking into account other quarks and leptons we obtain total widths and necessary branching ratios

$$
\begin{aligned}
& \Gamma_{t}\left(X^{ \pm}\right)=0.004393 \mathrm{GeV}: B R(j j)=0.0664 \\
& B R\left(W^{ \pm} \gamma\right)=0.900 ; \\
& \Gamma_{t}\left(X^{0}\right)=0.000511 \mathrm{GeV}: B R(j j)=0.714 ;
\end{aligned}
$$

where we associate a jet with each quark. Small total widths do not contradict to data [27] because the observed width of the enhancement corresponds to experimental resolution. One has also to bear in mind that real masses of neutral and charged $X$ may differ by few $\mathrm{GeV}$.

For estimation of $X$ production cross-sections we have to take into account that according to EW gauge invariance isotopic triplet $X^{a}$ necessarily interacts with gauge field $W^{a}$ and the interaction vertex is just the gauge one with the same coupling $g$

$$
\begin{aligned}
& \Gamma_{\mu \nu \rho}^{a b c}(p, q, k)=g \varepsilon^{a b c}\left(\Phi_{\kappa}(p, q, k) \kappa\left(g_{v \rho} k_{\mu}-g_{\rho \mu} k_{v}\right)+\right. \\
& \left.\Phi_{g}(p, q, k)\left(g_{\mu v}\left(q_{\rho}-p_{\rho}\right)-g_{v \rho} q_{\mu}+g_{\rho \mu} p_{v}\right)\right) ;
\end{aligned}
$$


where $\Phi_{\kappa, g}\left(p_{i}\right)$ are form-factors, which are equal to unity for $W$ momentum $k=0$ and other two momenta $p, q$ on the mass shell, $\kappa$ is the well-known parameter describing quadrupole interaction of a vector particle. In the present approximation $\kappa=0$. Effective total energy for partons' collisions at TEVATRON is around $330 \mathrm{GeV}$ that is essentially smaller than typical value (4.3). Thus, for estimates of cross-sections at TEVATRON we take $\Phi_{g}=1$. However at LHC energy is essentially larger and form-factors influence results. For the sake of estimates we will take for LHC

$$
\Phi_{g}(p, q, k) \simeq \Psi\left(k^{2}\right)^{2} ; \quad k^{2}=(1400 \mathrm{GeV})^{2} ; p^{2}=q^{2}=M_{X}^{2} \simeq 0 ;
$$

where we take for the energy of parton collisions one fifth of the total energy bearing in mind that point-like interaction leads to increasing cross-sections. In doing assumption (4.21) we take into account that in the first approximation $F_{g}$ is defined by one loop diagram with $\Psi$ in each vertex.

So taking into account all relevant interactions (4.5/4.17/4.20) we obtain the following estimates for cross-sections for energy $\sqrt{s}=1960 \mathrm{GeV}$

$$
\begin{aligned}
& \sigma\left(p \bar{p} \rightarrow W^{ \pm} X^{0}+\ldots\right)=1.86 p b ; \\
& \sigma\left(p \bar{p} \rightarrow W^{\mp} X^{ \pm}+\ldots\right)=1.71 p b ; \\
& \sigma\left(p \bar{p} \rightarrow Z X^{ \pm}+\ldots\right)=1.37 p b ; \\
& \sigma\left(p \bar{p} \rightarrow X^{0} X^{ \pm}+\ldots\right)=0.35 p b ; \\
& \sigma\left(p \bar{p} \rightarrow X^{\mp} X^{ \pm}+\ldots\right)=0.24 p b .
\end{aligned}
$$

Taking into account branching rations (4.19) we obtain for additional $W j j$ and $Z j j$ production in the region of enhancement the following estimate. We also divide cross-section for jet $-j e t$ production into two parts: with accompanying $\gamma$ and without $\gamma$

$$
\begin{aligned}
& \sigma\left(p \bar{p} \rightarrow W^{ \pm}+\gamma+2 j+\ldots\right)=0.26 p b \\
& \sigma\left(p \bar{p} \rightarrow W^{ \pm}+2 j+\ldots\right)=1.49 p b \\
& \sigma(p \bar{p} \rightarrow Z+2 j+\ldots)=0.13 p b
\end{aligned}
$$

Total cross-section for $W j j+W j j \gamma(1.75 p b)$ occurs to be considerably smaller than result [27] $\sigma(W j j)=4.0 \pm 1.2 p b$ whereas small value for $Z j j$ production quite agrees with [27] data. However just recently results of $D 0$ appear [25], which do not support large value for $\sigma(W j j)$ and give upper limit for cross-section under study $\sigma(W j j)<1.9 p b$ (95\% C.L.). As a matter of fact our result (4.23) evidently does not contradict both experimental results, because it differs from CDF number by less than two s.d..

The production of radial excitations $X_{i}$ may be compared with data on search of resonant $W W$ and $W Z$ production [32]. The results following from values of parameters $(4.15,4.16)$ are the following $\left(B_{i}=B R\left(X_{i} \rightarrow W W(Z)\right)\right)$

$$
X_{1}: \sigma B_{1}=0.15 p b ; \quad X_{2}: \sigma B_{2}=0.76 p b ; \quad X_{3}: \sigma B_{3}=1.64 p b .
$$

These results by no means contradict upper limits of work [32]. Note that $X_{i}$ production is accompanied by additional boson either $W$ or $Z$. Thus we predict effects in triple weak boson production: $W^{ \pm} W^{+} W^{-}, W^{+} W^{-} Z, W^{ \pm} Z Z$, which are connected with $X_{i}$ production. 
Process $p+p \rightarrow W^{ \pm}+\gamma+\ldots$ was studied at LHC for energy $\sqrt{s}=7 \mathrm{TeV}$ [30]. The results in comparison to SM calculations are the following

$$
\begin{aligned}
& \sigma\left(W^{ \pm} \gamma\right)=56.3 \pm 5.0(s t) \pm 5.0(s y) \pm 2.3(l u) p b \\
& \sigma\left(W^{ \pm} \gamma\right)_{S M}=49.4 \pm 3.8 p b
\end{aligned}
$$

The cross-sections for production of $X^{ \pm}$and $X^{0}$ at LHC are estimated to be

$$
\begin{aligned}
& \sigma\left(p p \rightarrow W^{ \pm} X^{0}+\ldots\right) \simeq 7.9 p b \\
& \sigma\left(p p \rightarrow W^{ \pm} X^{\mp}+\ldots\right) \simeq 4.7 p b ; \\
& \sigma\left(p p \rightarrow Z X^{ \pm}+\ldots\right) \simeq 5.7 p b ; \\
& \sigma\left(p p \rightarrow X^{\mp}+\ldots\right) \simeq 0.8 p b ; \\
& \sigma\left(p p \rightarrow X^{0} \ldots\right) \simeq 0.6 p b .
\end{aligned}
$$

These results are just estimation by an order of magnitude due to significant influence of formfactors in interactions $(4.5,4.20)$ at energy of LHC. In calculations we have used average values of form-factors in the region corresponding to the most probable $s_{\text {eff }}$ of partons: $\sqrt{s_{\text {eff }}} \simeq$ $700 \mathrm{GeV}$ [33]. Additional contribution from processes $(4.26)$ to $W^{ \pm} \gamma$ production reads

$$
\Delta \sigma\left(W^{ \pm} \gamma\right) \simeq 9.7 p b
$$

We see that here we also have no contradiction with data (4.25). Let us emphasize that this process is quite promising for checking of our scheme, because we not only predict additional contribution (4.27) but we insist that this additional contribution means production of narrow resonance $X^{ \pm}$ with mass around $145 \mathrm{GeV}$ which decays mostly to $W^{ \pm}+\gamma$.

Results of this section are obtained in work [34]. For calculations of cross-sections and decay widths the CompHEP package [35] was used.

\section{Conclusion}

To conclude we would emphasize, that albeit we discuss quite unusual effects, we do not deal with something beyond the Standard Model. We are just in the framework of the Standard Model. What makes difference with usual results are non-perturbative non-trivial solutions of compensation equations. With the present results we would draw attention to two important points. Firstly, the unique determination of gauge electro-weak coupling constant $g\left(M_{W}\right)$ and calculation of the $t$ - quark mass in close agreement with experimental values. These results strengthen the confidence in the correctness of applicability of Bogoliubov compensation approach to the principal problems of elementary particles theory. Secondly, we have seen, that non-perturbative contributions lead to prediction of experimental effects which are investigated at LHC and TEVATRON. These predictions at least do not contradict to the totality of data. More than that, there are some indications on agreement of several important effects with the predictions (the almost proved absence of Higgs scalar in the most popular region, a possible $W W$-bound state). 


\section{References}

[1] B.A. Arbuzov, Theor. Math. Phys., 140, 1205 (2004).

[2] B.A. Arbuzov, Phys. Atom. Nucl., 69, 1588 (2006).

[3] B.A. Arbuzov, M.K. Volkov and I.V. Zaitsev, Int. Journ. Mod. Phys. A, 21, 5721 (2006).

[4] B.A. Arbuzov, Phys. Lett. B, 656, 67 (2007).

[5] B.A. Arbuzov, M.K. Volkov and I.V. Zaitsev, Int. Journ. Mod. Phys. A 24, 2415 (2009).

[6] B.A. Arbuzov, Eur. Phys. J. C, 61, 51 (2009).

[7] N.N. Bogoliubov. Soviet Phys.-Uspekhi, 67, 236 (1959).

[8] N.N. Bogoliubov. Physica Suppl., 26, 1 (1960).

[9] N.N. Bogoliubov, Quasi-averages in problems of statistical mechanics. Preprint JINR D-781, (JINR, Dubna 1961).

[10] B.A. Arbuzov, A.N. Tavkhelidze and R.N. Faustov, Doclady AN SSSR, 139, 345 (1961).

[11] K. Hagiwara, R.D. Peccei, D. Zeppenfeld and K. Hikasa, Nucl. Phys. B, 282, 253 (1987).

[12] B.A. Arbuzov, Phys. Lett. B, 288, 179 (1992).

[13] LEP Electro-weak Working Group, arXiv: hep-ex/0612034v2 (2006).

[14] H. Bateman and A. Erdélyi, Higher transcendental functions. V. 1 (New York, Toronto, London: McGraw-Hill, 1953).

[15] Y. Nambu and G. Jona-Lasinio, Phys. Rev. 122, 345 (1961).

[16] Y. Nambu and G. Jona-Lasinio, Phys. Rev. 124, 246 (1961).

[17] M.K. Volkov and A. Radzhabov, Phys. Usp. 49, 551 (2006).

[18] Y. Nambu, Enrico Fermi Institute Report No 89-08, 1989.

[19] V.A. Miransky, M. Tanabashi and K. Yamawaki, Phys. Lett B 221, 177 (1989).

[20] W.A. Bardeen, C.T. Hill and M. Lindner, Phys. Rev. D 41, 1647 (1990).

[21] M. Lindner, Int. J. Mod. Phys. A 8, 2167 (1993).

[22] ATLAS Collaboration, arXiv: 1109.3357 [hep-ex] (2011).

[23] Tevatron Electroweak Working Group (CDF and D0 Collaborations), arXiv: 1007.3178 [hep-ex] (2010).

[24] B.A. Arbuzov and I.V. Zaitsev, Int. J. Mod. Phys. A, 26, 4945 (2011); arXiv:1107.5164 [hep-ph] (2011).

[25] V.M. Abazov et al. (D0 Collaboration), arXiv: 1106.1921 [hep-ex] (2011).

[26] T. Aaltonen et al. (CDF Collaboration), Phys. Rev. D, 82: 112001 (2010).

[27] T. Aaltonen et al. (CDF Collaboration), Phys. Rev. Lett., 106: 171801 (2011); arXiv: 1104.0699[hep-ex] (2011).

[28] V.M. Abazov et al. (D0 Collaboration), Phys. Rev. Lett., 102: 231801, 2009; arXiv: 0901.1887 [hep-ex] (2009). 
[29] The CMS Collaboration, arXiv:1102.5429 [hep-ex] (2011).

[30] D. Majuder (on behalf of the CMS Collaboration), arXiv: 1106.2829 [hep-ex] (2011).

[31] J.M. Campbell and R.K. Ellis, Phys. Rev. D, 60: 113006 (1999).

[32] V.M. Abazov et al. (D0 Collaboration), Phys. Rev. Lett., 107: 011801, 2011; arXiv: 1011.6278 [hep-ex] (2010).

[33] P.M. Nadolski et al, Phys. Rev. D, 78: 011801 (2008).

[34] B.A. Arbuzov and I.V. Zaitsev, arXiv: 1110.3137 [hep-ph] (2011).

[35] E.E. Boos et al. (CompHEP Collaboration), Nucl. Instr. Meth. A 534, 250 (2004). 